\title{
Síntesis mecanoquímica de un compuesto termoeléctrico nanocristalino $\left(^{(*)}\right.$
}

\author{
H. Rojas-Chávez* y **, F. Reyes-Carmona*** y D. Jaramillo-Vigueras*
}

\begin{abstract}
Resumen Se estudiaron las reacciones químicas en fase sólida durante la molienda mecánica de alta energía en condiciones cercanas a la atmosférica usando mezclas de polvos de $\alpha \mathrm{PbO}$ y Te de alta pureza. Se optó por esta vía de proceso para evitar las complejidades que surgen en otras técnicas tales como: fusión-crecimiento o sublimación de las especies reactivas, etc. El rastreo de la síntesis mecanoquímica (SM) muestra que conforme se incrementa el tiempo de molienda se disminuye el tamaño de partícula y se incrementa la cantidad de defectos estructurales. En el sistema $\alpha \mathrm{PbO}-\mathrm{Te}$, las fases metaestables obtenidas durante la molienda mecánica son las mismas que se forman durante el proceso convencional (fusión-crecimiento). Los polvos obtenidos por MS fueron caracterizados por difracción de rayos-X, microscopía electrónica de transmisión y microscopía electrónica de transmisión de alta resolución.
\end{abstract}

Palabras clave Reacciones químicas en fase sólida; Defectos estructurales; Sistema $\alpha \mathrm{PbO}-\mathrm{Te} ; \mathrm{PbTe}$.

\section{Mechanochemical synthesis of a nanocrystalline thermoelectric compound}

\begin{abstract}
Solid state chemical reactions were studied during high energy ball milling. Mechanosynthesis (MSY) of mixtures of high purity $\alpha \mathrm{PbO}$ and Te powders under atmospheric conditions were processed. This process was selected to minimize technical difficulties as those commonly found in conventional processes involving melting-growth or sublimation of starting materials. It has been found that as particle size is reduced, structural defects are increased and hence solid state chemical reactions are enhanced. In the MSY of $\alpha \mathrm{PbO}-\mathrm{Te}$, the phases formed are the same as those formed during conventional process (melt-growth). The alloyed powders obtained were characterized by X-ray diffraction, transmission electron microscopy and high resolution transmission electron microscopy.
\end{abstract}

Keywords Solid state chemical reaction; Structural defects; $\alpha \mathrm{PbO}-\mathrm{Te}$ system; PbTe

\section{INTRODUCCIÓN}

El teleruro de plomo $(\mathrm{PbTe})$ tiene una temperatura de fusión elevada, buena estabilidad química y baja presión de vapor. El $\mathrm{PbTe}$, es un material bien conocido por sus propiedades termoeléctricas en el intervalo de temperatura, de 500 a $900 \mathrm{~K}$. El PbTe se obtiene en forma policristalina y en monocristales. El PbTe policristalino, se procesa en tubos de silicio (al vacío y perfectamente sellados) que son calentados hasta $1200 \mathrm{~K}$, para asegurar la fusión, y posteriormente se enfría súbitamente el compuesto[1 y 2]. Existen otras técnicas alternativas para la obtención del $\mathrm{PbTe}$, tales como: síntesis hidrotérmica ${ }^{[3]}$, método Bridgman ${ }^{[4]}$, procesos al estado sólido ${ }^{[5]}$, por mencionar algunos.
Entre estas técnicas, la síntesis mecanoquímica (SM), a través de molienda mecánica (MM) de alta energía, ha ganado interés como técnica de procesamiento de no equilibrio, debido a su versatilidad y viabilidad para producir materiales meta-estables, aleaciones amorfas, materiales cuasi-cristalinos y nanocristalinos, compuestos intermetálicos y termoeléctricos ${ }^{[5-7]}$. Los compuestos intermetálicos se pueden fabricar a partir de polvos elementales que están sujetos a una serie de colisiones dentro de la MM ${ }^{[7}$ y 8]. Durante la MM se disminuye el tamaño de partícula y, en consecuencia, se incrementa su energía superficial. Cuando una partícula se fractura se rompe su estructura y se satura la estructura de defectos cristalinos, donde se espera que las superficies de las nuevas partículas se

(•) Trabajo recibido el día 15 de Abril de 2010 y aceptado en su forma final el día 24 de Septiembre de 2010.

* Instituto Politécnico Nacional (CIITEC) Cerrada CECATI s/n, Col. Sta. Catarina, Del. Azcapotzalco México, D.F. 02250.

** Instituto Politécnico Nacional (CICATA) Legaria 694, Col. Irrigación, Del. Miguel Hidalgo México, D.F. 11500.

*** Universidad Nacional Autónoma de México (F.Q.) Circuito de la Investigación Científica s/n, C.U. Del Coyoacán México, D.F. 04510. 
encuentren en un estado inestable. Tal inestabilidad de las superficies creadas les confiere una alta reactividad. Por ello, cuando las superficies recién creadas, o un volumen enriquecido en defectos cristalinos, son puestos en contacto con otra partícula, se espera que se induzca una reacción química. Por lo tanto, el producto de la reacción tendrá una composición diferente a la química de la partícula original ${ }^{[8]}$. Este tipo de reacciones al estado sólido, se efectúan solo cuando por la MM se alcanza una distribución de tamaños comprendidos en la escala nanométrica ${ }^{[8]}$. En contraste, la producción industrial del $\mathrm{PbTe}$ es un proceso muy largo que requiere el uso de elevadas temperaturas y vacío ${ }^{[1 \text { y } 2]}$. De allí que los parámetros de operación que se usan en la SM en condiciones cercanas a las atmosféricas, la convierten en una ruta alterna conveniente para producir materiales termoeléctricos policristalinos ${ }^{[1-5]}$.

En este estudio la MS de los polvos del sistema $\alpha \mathrm{PbO}-\mathrm{Te}$ vía $\mathrm{MM}$, se propone como un proceso mediante el cual se obtenga el $\mathrm{PbTe}$ nanométrico en condiciones atmosféricas.

Así mismo la MS del PbTe a partir de los polvos del sistema $\alpha \mathrm{PbO}$-Te en condiciones cercanas a las atmosféricas, puede reducir los costos de producción. Sin embargo, el objetivo principal de este trabajo, es investigar las reacciones químicas al estado sólido que ocurren durante las diferentes etapas de la MM, en condiciones cercanas a las atmosféricas.

\section{PROCEDIMIENTO EXPERIMENTAL}

\subsection{Preparación de las muestras}

El proceso de fabricación comenzó con polvos de $\alpha \mathrm{PbO}(5 \mu \mathrm{m})$ y $\mathrm{Te}(50 \mu \mathrm{m})$ (Sigma-Aldrich, > 99,9 \% masa), los cuáles fueron utilizados como materiales de inicio en toda la experimentación. Los polvos se mezclaron en la relación en masa 5:3 con la finalidad de formar el $\mathrm{PbTe}$. Las moliendas se realizaron en un molino SPEX 8000 mixer/mill utilizando contenedores de nylacero polimérico con caras hemisféricas para evitar las zonas muertas o de alto índice de segregación. El tiempo de molienda estuvo comprendido entre 0,083 y $4 \mathrm{~h}$ manteniendo constante la relación de peso bolas a peso muestra, 10:1. Las mezclas se vertieron en contenedores de nylacero y se mantuvo constante una atmósfera de aire. El medio de molienda que se utilizó fueron bolas de $\mathrm{ZrO}_{2}$ con diámetros de $10 \mathrm{~mm}$.

\subsection{Caracterización microestructural}

Para dar seguimiento a las transformaciones de fase de los polvos en función del tiempo de molienda, se realizó difracción de rayos-X (DRX) de las muestras obtenidas a diferentes tiempos de molienda. Los difractogramas fueron obtenidos con radiación $\mathrm{Cu}$ $\mathrm{K}_{\alpha}(\lambda=1,5418 \AA)$ a temperatura ambiente en un difractometro Bruker D8 Advance, con detector LynxEye. Las condiciones del barrido fueron: intervalo de $2 \theta$ desde 20 hasta $110^{\circ}$, paso de barrido de $0,020^{\circ}(2 \theta)$, debido a la pequeña cantidad de fase cristalizada en los polvos se utilizó un tiempo de conteo de $530 \mathrm{~s} \mathrm{paso}^{-1}$ y 0,3 en la apertura primaria.

Por medio de microscopía electrónica de transmisión (MET, JEOL JEM-1200 EX) y microscopía electrónica de transmisión de alta resolución (METAR, FEI JEM-1200 EX) se determinaron los cambios que ocurren en la microestructura de los polvos. Imágenes de campo claro y patrones de difracción de área seleccionada, fueron adquiridos de las muestras en las diferentes etapas de la molienda.

El tamaño promedio de partícula se determinó con el analizador de imágenes Sigma Scan Pro 5. Tanto las micrografías obtenidas por MET, como por METAR, fueron analizadas con dicho programa.

\section{RESULTADOS Y DISCUCIÓN}

\subsection{Caracterización por DRX}

\subsubsection{Análisis de los patrones de difracción}

Se analizaron los patrones de DRX correspondientes a las mezclas de polvos de $\alpha \mathrm{PbO}$ y Te para tiempos de molienda $\left(\mathrm{t}_{\text {mol }}\right)$ de 0,$083 ; 0,25$ y $0,50 \mathrm{~h}$, como se muestran en la figura 1 .

En la muestra con 0,083 h de molienda, se observa claramente la formación del compuesto intermetálico $\mathrm{PbTe}$, a partir de polvos de $\alpha \mathrm{PbO}$ y Te. Para $\mathrm{t}_{\text {mol }}>0,083 \mathrm{~h}$, los picos de difracción de las fases Te y $\propto \mathrm{TeO}_{2}$ desaparecen. En la muestra de 0,5 h de molienda, los picos de difracción del $\alpha \mathrm{PbO}$ desaparecen completamente y el patrón de difracción de rayos $\mathrm{X}$, corresponde exclusivamente a las fases $\mathrm{PbTe}$, $\mathrm{PbTeO}_{3}$ y $\alpha \mathrm{PbO}_{2}$. El punto más interesante es aquel que se presenta en las primeras etapas de la molienda $\left(t_{\text {mol }} \leq 0,50 \mathrm{~h}\right)$, en el que los polvos de $\alpha \mathrm{PbO}$ y Te se alean casi completamente dando lugar al compuesto nanocristalino $\mathrm{PbTe}$.

La presencia de los picos de difracción del PbTe en estas primeras etapas de la molienda, se racionaliza 


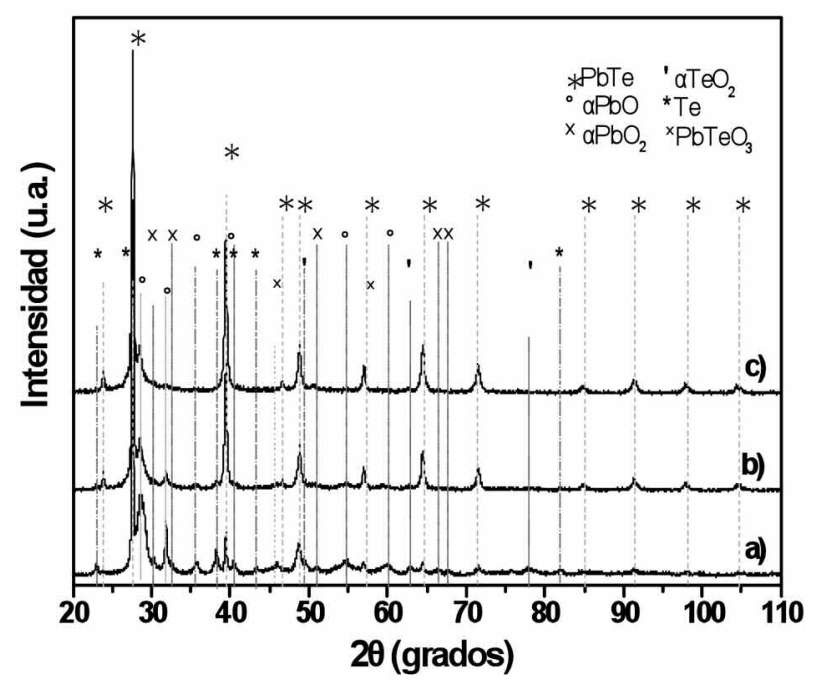

Figura 1. Difractograma de los polvos obtenidos a diferentes tiempos de molienda: 0,083, b) 0,25 y c) $0,50 \mathrm{~h}$.

Figure 1. Diffractogram of the powders obtained at different milling times: a) 0.083, b) 0.25 and c) $0.50 \mathrm{~h}$.

sólo con el diagrama de fases del Pb-Te ${ }^{[2}$ y 5$]$. Es importante mencionar que en dicho diagrama no existen otras fases intermedias. La formación del $\mathrm{PbTe}$, se explica también de acuerdo con el índice de Raynor $\left(\mathrm{I}_{\mathrm{R}}\right)$, para fases o compuestos únicos de composición $\mathrm{A}_{\mathrm{x}} \mathrm{B}_{(1-\mathrm{x})}$ dentro de un sistema binario:

$$
I_{R}=\frac{T_{m}\left(A_{x} B_{(1-x)}\right)}{x T_{m}(A)+(1-x) T_{m}(B)}
$$

En la ecuación (1): $T_{m}\left(A_{x} B_{(1-x)}\right)$; es la temperatura de fusión en $\mathrm{K}$ para el compuesto $\left(\mathrm{A}_{\mathrm{x}} \mathrm{B}_{(1-\mathrm{x})}\right)$ dentro del sistema binario. Cuando el $\mathrm{I}_{\mathrm{R}}>1$ es factible que se forme el compuesto $A_{x} B_{(1-x)}$. Por lo tanto, con los valores pertinentes para el sistema en cuestión, la probabilidad de formación del $\mathrm{PbTe}$ es elevada. Los valores altos del $I_{R}$ corresponden a sistemas binarios que ofrecen una gran facilidad en la formación de compuestos intermetálicos ${ }^{[9]}$. El valor del $I_{R}$ que se obtuvo para el PbTe fue: $I_{R}=1,810$.

Los resultados de DRX para las primeras etapas de la molienda $\left(\mathrm{t}_{\text {mol }} \leq 0,5 \mathrm{~h}\right)$ indican que la fase $\alpha \mathrm{PbO}$ se modifica hacia condiciones de mayor potencial de oxígeno, esto es, que cambia la relación estequiométrica tal que este óxido presenta una relación de $\mathrm{O}_{2}: \mathrm{Pb}>1$. La presencia de $\mathrm{O}_{2}(\mathrm{~g})$ de la atmósfera promueve estados de mayor oxidación tanto en el $\alpha \mathrm{PbO}$ como en el Te:

$$
\begin{gathered}
\alpha P b O+0.5 O_{2}(g) \rightarrow \alpha \mathrm{PbO}_{2} \\
\mathrm{Te}+\mathrm{O}_{2}(g) \rightarrow \alpha \mathrm{TeO}_{2}
\end{gathered}
$$

En la tabla 1, se resumen las diferentes fases detectadas por difracción de rayos $\mathrm{X}$ en las muestras obtenidas con $\mathrm{t}_{\mathrm{mol}} \leq 0,5 \mathrm{~h}$ a temperatura ambiente. De acuerdo con Oufkir ${ }^{[10]}$, la presencia del compuesto $\mathrm{PbTeO}_{3}$ es debida a la reacción que presenta el sistema $\alpha \mathrm{TeO}_{2}-\alpha \mathrm{PbO}$ :

$$
\alpha \mathrm{TeO}_{2}+\alpha \mathrm{PbO} \rightarrow \mathrm{PbTeO}_{3}
$$

Los óxidos de plomo con la relación de $\mathrm{O}: \mathrm{Pb}>1$ al estar en contacto íntimo con las partículas de telurio inducen la transición que dará lugar a la formación del PbTe:

$$
\begin{gathered}
\alpha \mathrm{PbO}+\mathrm{Te} \rightarrow \mathrm{PbTe}+1 / 2 \mathrm{O}_{2}(g) \uparrow \\
\alpha \mathrm{PbO}_{2}+\mathrm{Te} \rightarrow \mathrm{PbTe}+\mathrm{O}_{2}(g) \uparrow
\end{gathered}
$$

Tabla I. Fases detectadas por DRX de las muestras obtenidas a: $0,083,0,25$ y $0,50 \mathrm{~h}$ de molienda

Table I. Phases detected by XRD of the samples obtained: $0.083,0.25$ and $0.50 \mathrm{~h}$ milling time

\begin{tabular}{ccc}
\hline $\begin{array}{c}\text { Fases } \\
\text { presentes }\end{array}$ & ICDD & Nombre \\
\hline $\mathrm{PbTe}$ & $38-1435$ & Altaite \\
$\alpha \mathrm{PbO}$ & $85-1739$ & Litharge \\
$\alpha \mathrm{PbO}_{2}$ & $72-2440$ & Scrutinyte \\
$\mathrm{Te}^{2}$ & $36-1452$ & Telurio \\
$\alpha \mathrm{TeO}_{2}$ & $42-1365$ & Paratellurite \\
$\mathrm{PbTeO}_{3}$ & $39-0287$ & Óxido de plomo-telurio \\
\hline
\end{tabular}

La tabla 2, recoge las reacciones que se llevan a cabo durante las diferentes etapas de la molienda mecánica a temperatura ambiente del $\mathrm{PbTe}{ }^{[11]}$.

En la figura 2, los patrones para 1, 2 y $3 \mathrm{~h}$ de molienda muestran picos de difracción correspondientes al $\alpha \mathrm{PbO}_{2}$ y al PbTe. Para un $\mathrm{t}_{\text {mol }}=4 \mathrm{~h}$ desaparecen los picos de difracción del $\alpha \mathrm{PbO}_{2}$ y sólo queda una fase homogénea, que corresponde al $\mathrm{PbTe}$. 
SíNTESIS MECANOQUÍMICA DE UN COMPUESTO TERMOELÉCTRICO NANOCRISTALINO MECHANOCHEMICAL SYNTHESIS OF A NANOCRYSTALLINE THERMOELECTRIC COMPOUND

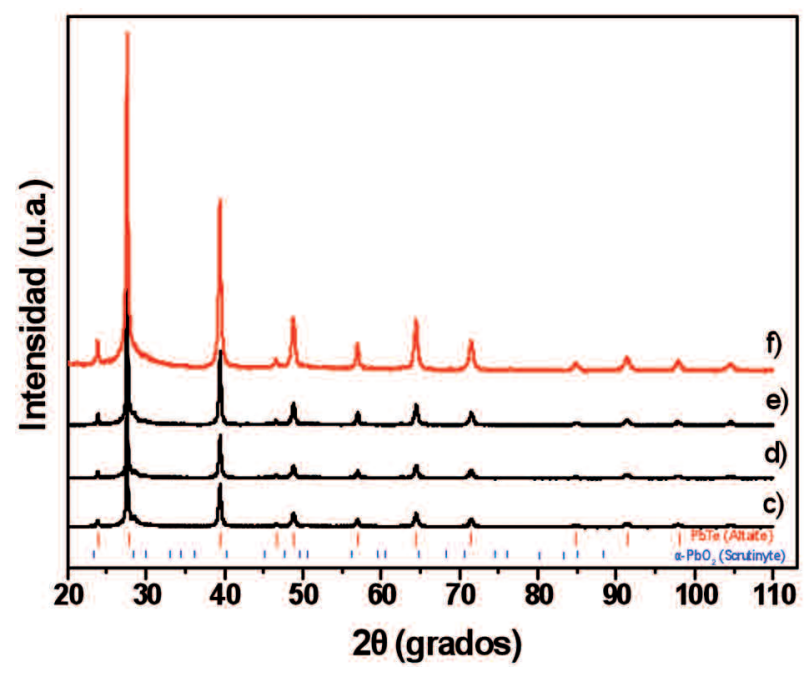

Figura 2. Difractograma de los polvos obtenidos a diferentes tiempos de molienda: a) 1 , b) 2 , c) 3 y d) $4 \mathrm{~h}$.

Figure 2. Diffractogram of the powders obtained at different milling times: a) 1, b) 2, c) 3 and d) $4 h$.

\subsection{Caracterización por microscopía electrónica}

\subsubsection{MET: Morfología y tamaño de partícula}

La figura 3, muestra micrografías típicas de MET de los polvos, en las diferentes etapas de molienda. Para una hora de molienda, en las micrografías se aprecia una dispersión de cristalitos de Te, los cuáles están distribuidos homogéneamente dentro de los óxidos de plomo, figura 3 (a y b). La mayoría de los cristalitos embebidos en los óxidos de plomo, tienen dimensiones que están en el rango de 5 a $10 \mathrm{~nm}$; en esta etapa de la molienda se produce un crecimiento aparente del tamaño de partícula (>50 nm), debido a la aglomeración de éstas. Después de $2 \mathrm{~h}$ de molienda el tamaño promedio de partícula se redujo a menos de $30 \mathrm{~nm}$. De esta forma, conforme incrementa el tiempo de molienda $\left(\mathrm{t}_{\mathrm{mol}} \geq 3 \mathrm{~h}\right)$, el tamaño de partícula disminuye hasta $\sim 20 \mathrm{~nm}$, ver figura $3 \mathrm{c}$, y se mantiene inalterable pese a que se sigan formando aglomerados, (Fig. $3 \mathrm{~d}$ ).

Los patrones de difracción de área seleccionada, obtenidos de áreas $\sim 1 \mu \mathrm{m}$ de diámetro, muestran una transición gradual de puntos dispersos, figura 3 (a y b), a una estructura de anillos continuos, conforme se incrementa el tiempo de molienda (insertos en la parte superior derecha de la figura 3 ( c y d). Todos
Tabla II. Reacciones presentes durante las diferentes etapas de la molienda mecánica

Table II. Reactions present during the different ball milling stages

\begin{tabular}{cc}
\hline Reacción & $\Delta$ G (kJ/mol) \\
\hline$\alpha \mathrm{PbO}+1 / 2 \mathrm{O}_{2} \rightarrow \alpha \mathrm{PbO}_{2}$ & $-27,248$ \\
$\mathrm{Te}+\mathrm{O}_{2} \rightarrow \alpha \mathrm{TeO}_{2}$ & $-270,867$ \\
$\mathrm{PbO}+\alpha \mathrm{TeO}_{2} \rightarrow \mathrm{PbTeO}_{3}$ & $-308,1556$ \\
$\mathrm{PbO}+\mathrm{Te} \rightarrow \mathrm{PbTe}+1 / \mathrm{O}_{2} \uparrow$ & $+121,684$ \\
$\mathrm{PbO}_{2}+\mathrm{Te} \rightarrow \mathrm{PbTe}+\mathrm{O}_{2} \uparrow$ & $+148,932$ \\
\hline
\end{tabular}

los anillos de los patrones de difracción de área seleccionada, son los correspondientes a la fase de $\mathrm{PbTe}$.

\subsubsection{METAR: Tamaño de partícula y defectos microestructurales}

Para revelar información de la estructura a nivel atómico, se realizó METAR. En la figura 4 se observan imágenes de METAR de las nanopartículas obtenidas en la SM. El tamaño de partícula para la muestra, con 1 h de molienda, está en el rango comprendido entre 10 y $20 \mathrm{~nm}$. Con este tiempo de molienda predominan las caras de los planos (200), cuya distancia interatómica es de 0,32325 nm, ver figura 4 a). En la figura $4 \mathrm{~b}$ ), se aprecia que las reacciones químicas al estado sólido y el posterior crecimiento de las partículas, se presenta a través de la gran cantidad de límites de grano, formados durante el proceso de MM. En la figura $4 \mathrm{c}$ ), se muestra una zona con una alta concentración de dislocaciones, causadas por el proceso de MM. De donde se espera que este tipo de defectos, al igual que los límites de grano, sirvan para promover las reacciones químicas al estado sólido con las cuáles se favorecerá la formación del PbTe. A diferencia de los procesos convencionales, en la microestructura del $\mathrm{PbTe}$ obtenida en la $\mathrm{SM}$, deben esperarse que las dislocaciones den lugar a una diferencia estructural significativa, ya que estas zonas de alta densidad de dislocaciones incrementan las propiedades termoeléctricas ${ }^{[12 \text { y 13] }}$. La figura $4 \mathrm{~d}$ ), muestra un área en donde se tienen dos tipos de distancia interatómica del PbTe, 0,32325 nm (200) y 0,22845 (220). Por medio METAR se puede apreciar la presencia de partículas, cuyo tamaño está comprendido entre 5 y $6 \mathrm{~nm}$, de esta manera los aglomerados que se mencionaron en la sección anterior, no solo estarían compuestos por partículas $\sim 20 \mathrm{~nm}$ sino también por otras más pequeñas. 

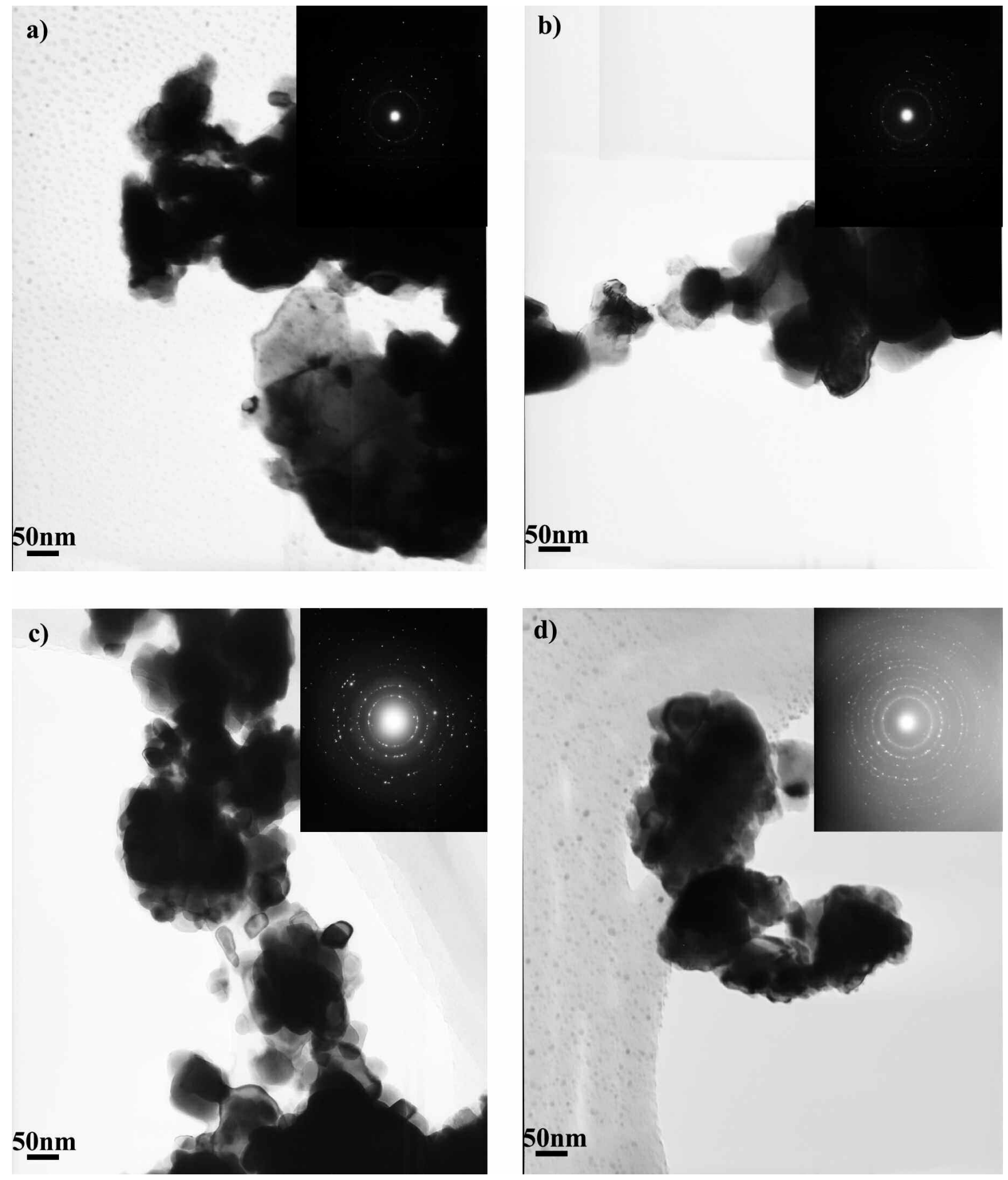

Figura 3. Micrografías obtenidas por MET de los polvos obtenidos a diferentes tiempos de molienda: a) $1 \mathrm{~h}$; b) $2 \mathrm{~h}$; c) $3 \mathrm{~h}$ y d) $4 \mathrm{~h}$.

Figure 3. TEM micrographs of the powders obtained at different milling times: a) $1 h$; $b) 2 h$;c) 3 $h$ and d) $4 h$.

\section{CONCLUSIONES}

- La síntesis del teleruro de plomo (PbTe), se puede obtener por molienda mecánica de alta ener- gía en un molino SPEX, utilizando condiciones cercanas a las atmosféricas y cuando se usa una mezcla inicial de $\alpha \mathrm{PbO}$ y Te en la relación en masa 5:3. Por esta vía, en tanto se parta de especies 
SÍNTESIS MECANOQUIIMICA DE UN COMPUESTO TERMOELÉCTRICO NANOCRISTALINO MECHANOCHEMICAL SYNTHESIS OF A NANOCRYSTALLINE THERMOELECTRIC COMPOUND
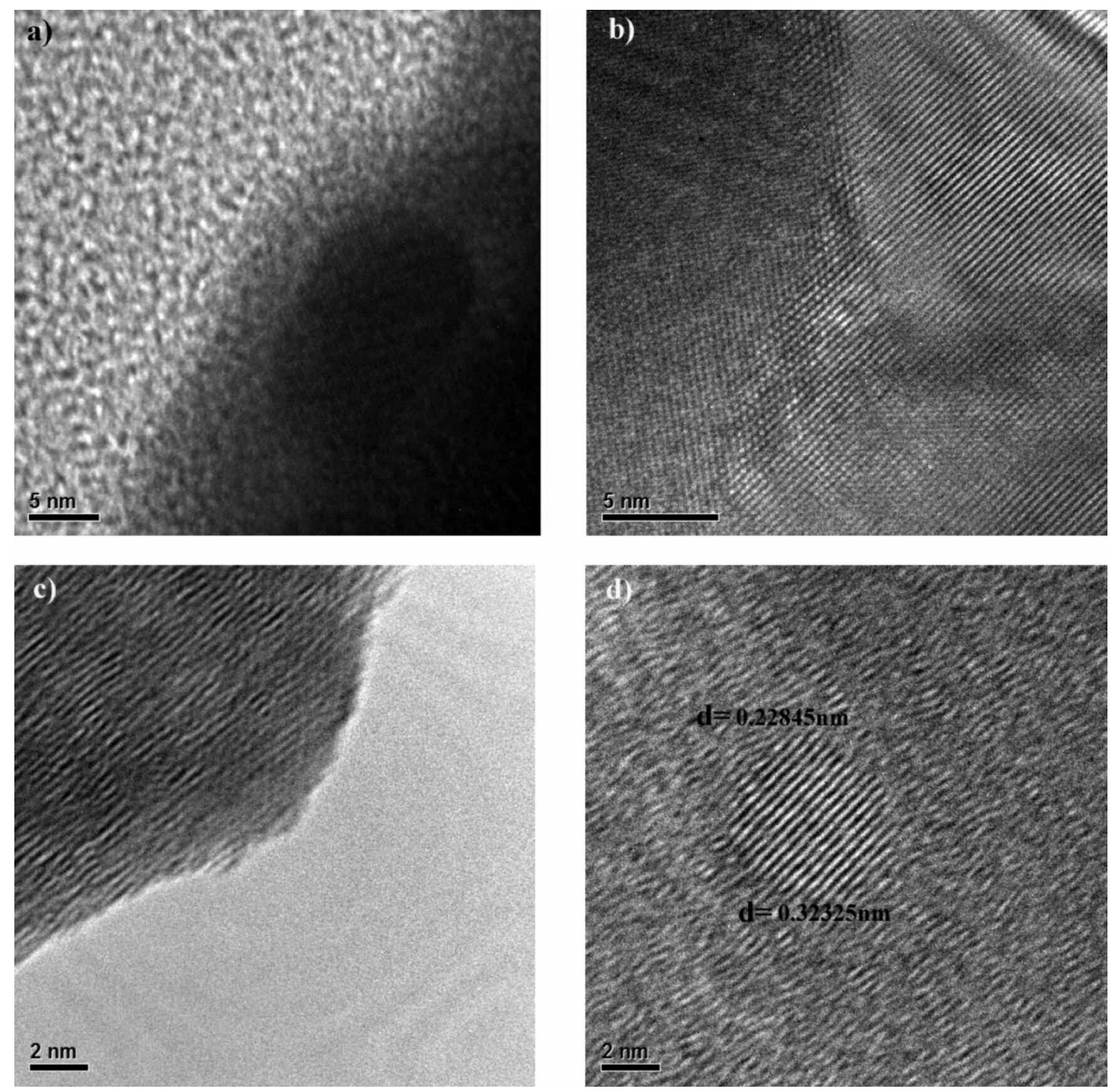

Figura 4. Micrografías obtenidas por METAR de los polvos obtenidos a diferentes tiempos de molienda: a) $1 \mathrm{~h}$; b) $2 \mathrm{~h}$; c) $3 \mathrm{~h}$ y d) $4 \mathrm{~h}$.

Figure 4. HRTEM micrographs of the powders obtained at different milling times: a) $1 \mathrm{~h}$; b) $2 h$;c) $3 h$ and d) $4 h$.

puras; este compuesto no presentará contaminación de ningún tipo.

- Después de 4 h de molienda mecánica de alta energía, bajo condiciones cercanas a las atmosféricas y con los parámetros de operación antes descritos, se obtuvo la SM del PbTe en forma nanocristalina $(\sim 20 \mathrm{~nm})$.

- Las colisiones que se dan durante la MM proveen de un alto grado de deformación de la red cristalina induciendo zonas con alta densidad de dislocaciones, y rápidamente refinan el tamaño de partícula. La gran cantidad de energía que se genera durante la MM promueve las diferentes reacciones al estado sólido para formar el $\mathrm{PbTe}$. El tiempo de molienda necesario para obtener la $\mathrm{SM}$ del $\mathrm{PbTe}$ a partir del sistema $\alpha \mathrm{PbO}-\mathrm{Te}$, es mucho menor que el reportado por otros autores, para la obtención del PbTe utilizando el sistema $\mathrm{Pb}-\mathrm{Te}$.

\section{Agradecimientos}

Los autores reconocen y agradecen al: M. en C. M. Aguilar-Franco (IF-UNAM), M. en C. J.M. JuárezGarcía (CENAM), V. Garibay-Febles (IMP) e Ing. P. Castillo (UAM-I) por su apoyo técnico. Uno de los autores (H. Rojas-Chávez) agradece también la 
extensión del soporte económico parcial recibido por el CONACyT (México).

\section{REFERENCIAS}

[1] J. Schilz, M. Riffel, K. Pixius, H.J. Meyer, Powder Technol. 105 (1999) 149-154.

[2] O. Tae, C. Jae, H. Dow, Scripta Metall. Mater. $32 / 4$ (1995) 595-600.

[3] T.J. Zhu, Y.Q. Liu y X.B. Zhao, Mater. Res. Bull 43 (2008) 2.850-2.854.

[4] V. Muñoz, A. Lasbley, S. Klotz, R. Triboulet, J. Crystal Growth 196 (1999) 71-76.

[5] N. Bouad, R.M. Marin y J.C. Tédenac, J. Alloys Compd. 297 (2000) 312-318.

[6] H. Rojas y D. Jaramillo., Superficies y Vacio 21/ 1 (2008) 11-15.
[7] H. Rojas-Chávez, F. Reyes-Carmona, G. Plascencia y D. Jaramillo-Vigueras, Mater. Sci. Forum 644 (2010) 69-74.

[8] M. Hosokawa, K. Nogi, M. Naito y T. Yocoyama, Nanoparticle Technology Handbook, ELSEVIER, The Netherlands, 2007.

[9] R. Ferro y A. Saccone, Intermetallic Chemistry, Pergamon-Elsevier, Great Britain, 2008.

[10] A. Oufkir, M. Dutreilh, P. Thomas, J.C. Champarnaud-Mesjard, P. Marchet y B. Frit, Mater. Res. Bull. 36 (2001) 693-703.

[11] I. Barin, Thermochemical data of pure substances, $3^{\text {rd }}$ Ed., Weinheim, New York, VCH, 1995.

[12] A. Farghalli, A. Mohamed y Y. Xun, Mater. Sci. Eng. A 354 (2003) 133-139.

[13] H. Beyer, J. Nurnus, H. Böttner, A. Lambrecht, E. Wagner y G.Bauer, Phys. E 13 (2002) $965-$ 968. 